\title{
AUGMENTING TUMOR SENSITIVE MATCHING FLOW TO IMPROVE DETECTION AND SEGMENTATION OF OVARIAN CANCER METASTASES WITHIN A PDE FRAMEWORK
}

\author{
Jianfei Liu ${ }^{1}$, Shijun Wang ${ }^{1}$, Marius George Linguraru², Jianhua Yao ${ }^{1}$, Ronald M. Summers ${ }^{1}$ \\ 1. Imaging Biomarkers and Computer-Aided Diagnosis Laboratory, Radiology and Imaging \\ Science, National Institutes of Health Clinical Center, Bethesda, MD 20892 \\ 2. Sheikh Zayed Institute for Pediatric Surgical Innovation, \\ Children's National Medical Center, Washington, DC 20010
}

\begin{abstract}
The detection and segmentation of ovarian cancer metastases have potentially great clinical impact on women's healthcare. We recently developed a tumor sensitive matching flow (TSMF) technique to locate metastases by juxtaposing the roles of matching and classification within a PDE framework. This paper further augments the TSMF approach by integrating 1) shape index to measure metastasis-caused deformation, 2) Gaussian mixture model to describe metastasis intensity distribution, 3) total variation (TV) flow to enhance metastasis regions, and 4) TSMF vector displacements to control the amount of level-set propagation. The method was validated on contrast-enhanced CT data from 30 patients, of which 15 have 37 metastases in total. The true positive rate was $87 \%$ compared to $76 \%$ in our earlier work. Moreover, the false positive rate per patients was dropped to 1.1 from 4.2 in our earlier work. The metastasis segmentation achieved a Dice coefficient of $80.0 \pm 7.2 \%$. All these experimental results demonstrated that shape index, Gaussian mixture model, TV flow, and TSMF-constrained level set propagation substantially improve the accuracy of metastasis detection and segmentation.
\end{abstract}

Index Terms - Ovarian cancer metastases, tumor sensitive matching flow, tumor segmentation

\section{INTRODUCTION}

Ovarian cancer is a deadly disease, in large measure because it metastasizes frequently, in as many as $75 \%$ of women in one study [1]. Detecting and segmenting ovarian cancer metastases have potentially great impact on improving the prognosis and treatment of women with ovarian cancer.

The Random distribution and weak boundaries of metastases introduce substantial challenges to their detection and segmentation. Machine learning techniques are commonly used to establish CAD systems for CT image detection of tumors within human organs [2,3,4,12]. However, we focus on detecting metastases external to and attached to organs. We propose a detection algorithm with minimum need for training to avoid time-consuming collection of training data. Metastases can be alternatively located by comparing patient images to atlas images from healthy individuals because metastases often cause organs to deform. For this purpose, optical flow methods are known to measure visual motion or shape variance between two images $[5,6]$. Unfortunately, these flow field methods fail to reliably find metastases because individual differences dominate the shape variation.

We recently developed a tumor sensitive matching flow (TSMF) technique to detect metastases [7]. The key contribution was the integration of a metastasis-likelihood function into a PDE-based shape comparison framework. The likelihood function evaluated the existence of metastases in local image regions and artificially controlled the flow computation. TSMF highlights metastasis regions in the flow field while dampening all other variance. Metastases can thus be detected and segmented by searching large TSMF vectors.

This paper improves the TSMF approach by incorporating shape index and Gaussian mixture models. The augmented TSMF is more sensitive to metastases and enhances the accuracy of detection. We also use total variation (TV) flow [8] to diffuse image regions with metastases while preserving their boundaries in order to alleviate the over-segmentation issue. Moreover, a level set algorithm used to segment metastases is optimally controlled by the displacements of TSMF vectors. The validation on datasets from 30 patients demonstrated that the augmented TSMF algorithm significantly improved the accuracy of ovarian cancer metastasis detection and segmentation.

\section{METHODOLOGY}

Our ovarian cancer metastasis detection and segmentation consists of shape descriptor construction, TSMF computation, seed point determination, and metastasis segmentation, as illustrated in Fig. 1. This section will particularly elaborate on the enhancements to this detection framework. Some of the implementation details are presented in our earlier work [7]. 


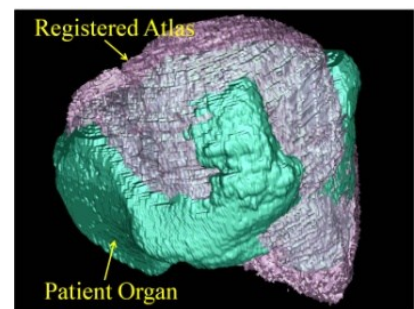

(a)

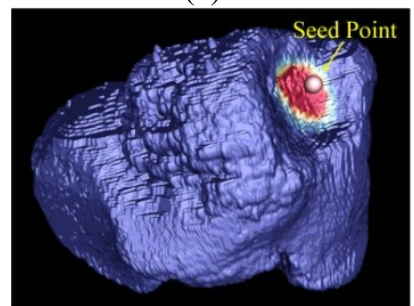

(c)

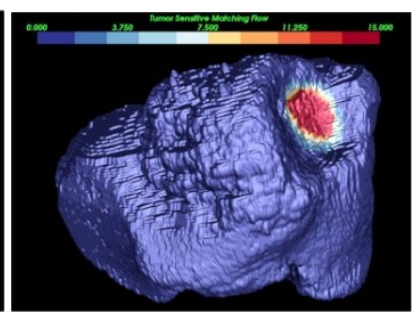

(b)

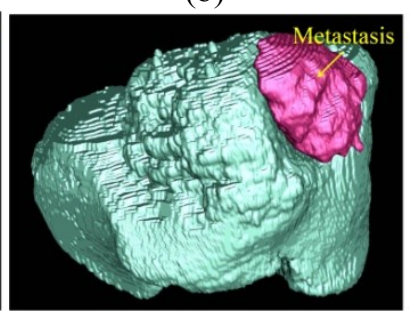

(d)
Figure 1: Process of ovarian cancer metastasis detection and segmentation. (a) Patient organ and registered atlas from shape descriptor construction (overlaid), (b) TSMF field where blue to red represents small to large flow vectors, (c) seed point determined from TSMF field, and (d) the segmented metastasis in red.

\subsection{Shape descriptor construction}

This step first registers a reference CT image to the current patient image $\left(I_{p}\right)$ and generates a registered reference image $\left(I_{a}\right)$. The image pair, $I_{p}$ and $I_{a}$, is used to measure the intensity difference between the current patient and the healthy individual. The liver and spleen are then automatically segmented from the patient image using the method in [13]. Image registration is again performed between a probabilistic atlas and the segmented organs, as shown in Fig. 1(a), followed by distance transforms [9]. We obtain another image pair, organ distance field $\left(D_{p}\right)$ and atlas distance field $\left(D_{a}\right)$, serving for shape description.

\subsection{Tumor sensitive matching flow (TSMF) computation}

TSMF aims to measure the shape variance between the patient organ and the atlas to identify metastases. Embedding the two image pairs obtained from the previous step into a PDE framework leads to

$$
\begin{aligned}
E(\vec{u}) & =\iiint_{(x, y, z) \in R^{3}} \Psi\left(\left(I_{a}\left(x+u_{x}, y+u_{y}, z+u_{z}\right)-I_{p}(x, y, z)\right)^{2}\right) \\
& +\alpha C(x, y, z) \underbrace{\Psi\left(\left(\nabla I_{a}\left(x+u_{x}, y+u_{y}, z+u_{z}\right)-\nabla I_{p}(x, y, z)\right)^{2}\right)}_{\text {Intensity Constancy }} \\
& +\beta C(x, y, z) \underbrace{\Psi\left(\left(D_{a}\left(x+u_{x}, y+u_{y}, z+u_{z}\right)-D_{p}(x, y, z)\right)^{2}\right)}_{\text {Gradient Constancy }} \\
& +\gamma \underbrace{\Psi\left(\left|\nabla u_{x}\right|^{2}+\frac{\left|\nabla u_{y}\right|^{2}}{\underbrace{}_{\text {Flow Smoothness }}-\frac{\left|\nabla u_{z}\right|^{2}}{\Psi})} d x d y d z\right.}_{\text {Distance Constancy }}
\end{aligned}
$$

Here, $\vec{u}=\left(u_{x}, u_{y}, u_{z}\right)$ represents the TSMF vector. $\alpha=50$, $\beta=5000$, and $\gamma=100$ are constants to balance different components, and $\Psi\left(x^{2}\right)=\sqrt{x^{2}+\varepsilon^{2}}, \varepsilon=0.001$ is a modified L1 norm. $C(x, y, z)$ is a metastasis-likelihood function for evaluating metastasis existence at point $(x, y, z)$, which is the key factor that enables TSMF (computed from Eq. 1) to find metastases.

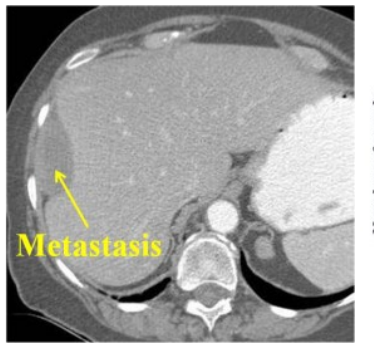

(a)

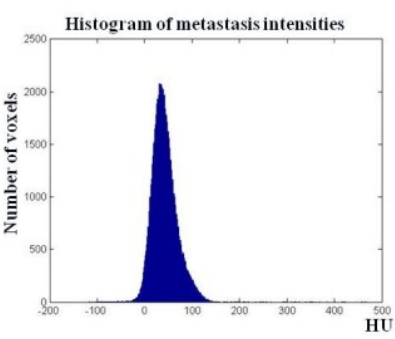

(b)
Figure 3: Intensity distribution of the metastasis.

The definition of $C(x, y, z)$ is based on the observation that the metastasis intensity distribution is similar to a Gaussian function (see Fig. 3), and the motivation that we detect external metastases attached to organs. We assume $\mu_{m}$ and $\sigma_{m}$ to be the mean and standard deviation of the CT attenuation (voxel intensities) of the metastasis. $C(x, y, z)$ is a pieceswise function and given by

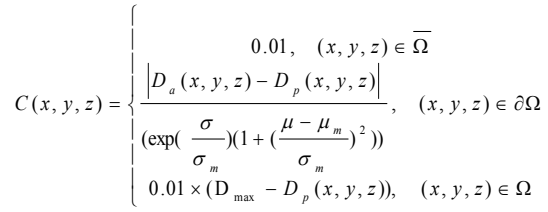

where $\Omega$ and $\bar{\Omega}$ are the segmented organs and non-organ regions in the patient image, and $\partial \Omega$ is the organ boundary. $\mathrm{D}_{\max }$ is the largest distance value in the patient distance field, and $\mu$ and $\sigma$ are the mean intensity and standard deviation of adjacent voxels in $\bar{\Omega}$. Eq. 2 indicates that the likelihood of the metastasis existence remains a small value in the non-organ regions and also decreases inside the organ. The likelihood significantly increases if the local organ boundary has large shape change (measured by the numerator) or the intensity values within the metastasis are more homogeneous (denominator).

Using the Euler-Lagrange equation to minimize Eq. 1 yields TSMF, and TSMF vectors are magnified at metastasis regions thanks to $C(x, y, z)$. Metastases can thus be identified by searching for large TSMF vectors. However, the numerator in Eq. 2 fails to reliably classify the cup shape caused by metastases and produces many false positives, as illustrated in the third row of Fig. 4. In addition, a single Gaussian function is insufficient to describe wide metastasis intensity ranges. To address these issues, we study shape index and Gaussian mixture models to improve $C(x, y, z)$.

Shape Index. Letting $\kappa_{1}<\kappa_{2}$ be the principal curvatures of the organ surface, the shape index [10] is defined as 
$S\left(\kappa_{1}, \kappa_{2}\right)=-\frac{2}{\pi} \operatorname{atan}\left(\frac{\kappa_{1}+\kappa_{2}}{\kappa_{2}-\kappa_{1}}\right)$

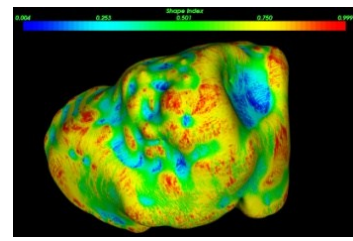

Figure 2: Shape index on the liver surface.
Fig. 2 illustrates the shape index mapped on the liver surface. Blue to red colors represent small to large shape index values. We are interested in cup shapes on the liver surface because metastases often push against the organ causing deformation. Shape index in Fig. 2 accurately tracks metastasis areas because their blue regions correspond to cup shapes. Therefore, shape index is a discriminative feature to enhance $C(x, y, z)$.

Gaussian mixture model. In order to reduce individual variability, we choose six representative metastases from six patients not used in our validation experiments to compute a Gaussian mixture model for describing the intensity distribution of the metastasis, which is defined as

$P_{\text {metastasis }}=\sum_{i=1}^{6} \frac{1}{\sqrt{2 \pi} \sigma_{i}} \exp \left(-\frac{\left(I-\mu_{i}\right)^{2}}{\sigma_{i}^{2}}\right)$

where $\mu_{i}$ and $\sigma_{i}$ are the mean intensity and standard deviation of $i$-th representative metastasis.

Exploiting the shape index and Gaussian mixture model, the center equation of $C(x, y, z)$ in Eq. 2 is reformulated as

$$
\frac{\left|D_{a}(x, y, z)-D_{p}(x, y, z)\right|}{\left(\sum_{i=1}^{6} w_{i} \exp \left(\frac{\sigma}{\sigma_{i}}\right) / \sum_{i=1}^{6} w_{i}\right)\left(1+\sum_{i=1}^{6} w_{i}\left(\frac{\mu-\mu_{m}}{\sigma_{m}}\right)^{2} / \sum_{i=1}^{6} w_{i}\right)\left(1+S\left(\kappa_{1}, \kappa_{2}\right)\right)}
$$

Here, $w_{i}=1$ in our implementation. Note that the shape index term is placed in the denominator since small shape index values correspond to the cup shape caused by metastases. The other two terms in the denominator measure the homogeneity and intensity difference between the metastasis and the current image region. $C(x, y, z)$ yields large values if the current image region contains metastases. Otherwise, it will suppress the TSMF flow.

Finally, TSMF is augmented by using the enhanced $C(x, y, z)$ and minimized by the Euler-Lagrange equation. Fig. 1(b) shows the augmented TSMF result, where the metastasis regions are highlighted by large TSMF vectors.

\subsection{Metastasis segmentation}

Metastasis segmentation involves two main steps, seed point determination and fast-matching segmentation.

Seed point determination starts with establishing connection graphs on the organ surface. TSMF vectors can measure metastasis-caused shape variance among organ surface. Surface points with TSMF vector magnitude larger than $10 \mathrm{~mm}$ are selected as we are only interested in detecting metastases larger than $10 \mathrm{~mm}$. Each connection graph is then subdivided into small subgraphs. Each subgraph has approximately 100 vertices $(10 \mathrm{~mm} \times 10 \mathrm{~mm}$ area) in order to detect metastases larger than $10 \mathrm{~mm}$. Finally, the center point of each sub-graph is chosen as the seed point, as shown in Fig. 1(c).

Total-variation (TV) flow [8] is used to remove heterogeneity in the metastasis while preserving image boundaries. Seed points are imported into the fast-matching algorithm [11]. To control over-segmentation, we set the iteration number as $2.5|\vec{u}|$ because TSMF vectors indicate local deformation caused by metastases. Finally, the metastasis is segmented as depicted in Fig. 1(d).

\subsection{Validation dataset}

The augmented TSMF algorithm was validated on abdominal contrast-enhanced CT images from 30 patients. 15 patients had at least one metastasis, and the total number of metastases was 37. They were all labeled by an experienced radiologist and used as the ground-truth. Thirtytwo of them were attached to liver, and the remaining four to the spleen. Their size range was $4.0-49.9 \mathrm{~mm}$. The CT image slice thickness range was $2.0-2.5 \mathrm{~mm}$. Retrospective analyses of these images were approved by our Institutional Review Board.

We chose true positive (TP) rate and false positive (FP) per patient to evaluate the accuracy of the metastasis detection. We also employed the Dice coefficient (DC) to measure segmentation accuracy.

\section{EXPRIMENTAL RESULTS}

The true positive rate of the augmented TSMF was $87 \%$ and the false positive per patient was 1.1 , while they were $76 \%$ and 4.2 by using our previous approach [7]. Note that three metastases were due to small size (less than $10 \mathrm{~mm}$ ) and the remaining two because of minor deformation. Metastases larger than $10 \mathrm{~mm}$ are important as they are clinically useful for the follow-up treatment. The average Dice coefficient of the segmented tumors was $80.0+/-7.2 \%$ with augmented TSMF, while it was $62.0+/-6.1 \%$ with the original TSMF.

Fig. 4 compares metastasis detection on four patients by using original and augmented TSMF. The first patient had three metastases. Metastasis (A) was missed in both methods because it was floating close to the liver. Metastasis (B) was missed in the original approach, but detected by the augmented TSMF because the augmented TSMF is equipped with the shape index and Gaussian mixture model to accurately determine the existence of metastases in local image regions. Moreover, metastasis (C) was over-segmented by the original TSMF while it was similar to the annotated correspondence using the augmented TSMF. The patient in the second column had five metastases including one attached to the spleen, labeled (E). The original TSMF missed (E) and produced a couple of false positives near the liver. The augmented TSMF 
detected all metastases, with only one false positive. In addition, metastasis (D) is more accurately segmented by the augmented TSMF. The two patients in the third and fourth columns had no metastases. The original TSMF generated multiple false positives at image regions (F-I), while the augmented TSMF only produced one. The false positive $(\mathrm{F})$ is on the gallbladder, which is a typical false positive because the gallbladder has similar intensity to some low attenuation metastases.
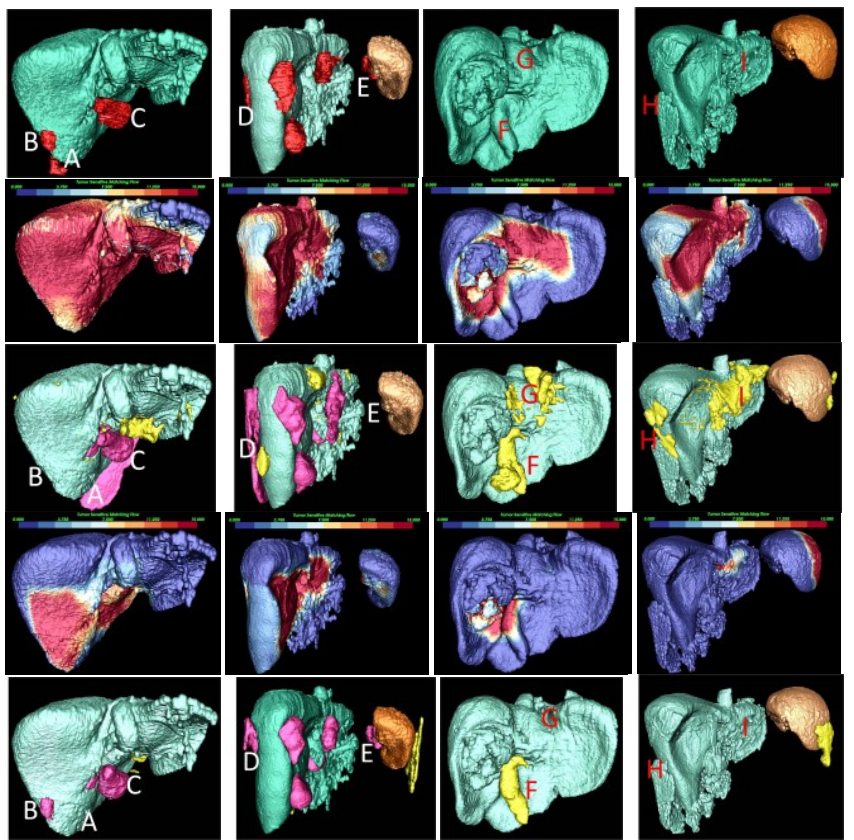

Figure 4: Comparison of segmented metastases on four patients using original and augmented TSMF. Two patients with metastases are illustrated in the left two columns, and two patients without metastases in the right two columns. First row: Ground-truth metastases (red) attached to liver (green) or spleen (brown). They are void in the patients without metastases; Second row: original TSMF; Third row: segmented metastases from original TSMF, where true detections are shown in red and false detections in yellow; Fourth row: augmented TSMF; Fifth row: segmented metastases from augmented TSMF.

\section{CONCLUSIONS}

In this work, we augmented the PDE framework of tumor sensitive matching flow for ovarian cancer metastasis detection and segmentation by embedding 1) shape index, 2) Gaussian mixture model, 3) total variation flow, and 4) TSMF-constrained level set propagation. The integration of shape index and Gaussian mixture model better describes the likelihood of metastasis existence in the current image region, and TV-flow and TSMF-constrained metastasis segmentation help to reduce metastasis over-segmentation.

The augmented approach was validated on 30 patients, with a total of 37 metastases. The augmented TSMF improved the true positive rate from $76.0 \%$ to $87.0 \%$, while dropping the false positive per patient from 4.2 to 1.1 . The average Dice coefficient of the segmented tumors was also enhanced from $62.0 \%$ to $80.0 \%$. Results demonstrated that the augmented TSMF approach was more effective for metastasis detection and segmentation.

\section{ACKOWLEDGEMENT}

This work was supported by the Intramural Research Program of the National Institutes of Health, Clinical Center.

\section{REFERENCES}

[1] S. Memarzadeh, and S.B. Roberts, "Advance in the management of epithelial ovarian cancer," The Journal of Reproductive Medicine, vol. 46, pp. 621-629, 2001.

[2] D. Smeets, D. Loeckx, and B. Stijnen, "Semi-automatic level set segmentation for liver tumors combining a spiral techniques with supervised fuzzy pixel classification," Medical Image Analysis, vol. 14, no.1, pp. 13-20, 2010.

[3] R.M. Summers, "Computed tomographic virtual colonoscopy computer-aided polyp detection in a screening population," Gastroenterology, vol. 129, pp. 1832-1844, 2005.

[4] M. Bilello, and S.B. Gokturk, "Automatic detection and classification of Hypodense hepatic lesions on contrast-enhanced venous-phase ct," Medical Physics, vol. 31, pp. 2584-2593, 2004.

[5] J. Liu, and K.R. Subramanian, and T.S. Yoo, "Temporal volume flow: an approach to tracking failure recovery," in SPIE Medical Imaging, Lake Buena Vista, Florida, 2011.

[6] T. Brox, A. Bruhn, N. Papenberg, and J. Weickert, "High accuracy optical flow estimation based on a theory for warping," in ECCV, vol. 3024, pp. 25-36, 2004.

[7] J. Liu, S. Wang, M.G. Linguraru, and R.M. Summers, "Tumor Sensitive Matching Flow: An Approach for Ovarian Cancer Metastasis Detection and Segmentation," in MICCAI Workshop on Abdominal Imaging, vol. 7061, pp. 265-273, 2012.

[8] T. Brox, and J. Weickert, "A TV flow based local scale estimate and its application texture discrimination," Journal of Visual Communication and Image Representation, vol. 17, no. 5, pp. 1053-1073, 2006.

[9] C.R. Maurer Jr., R. Qi, and V. Raghavan, "A linear time algorithm for computing exact Euclidean of distance transform of binary images in arbitrary dimension," IEEE PAMI, vol. 25 , no. 2 , pp. 265-270, 2003.

[10] J. Koenderink, and A. van Doorn, "Surface shape and curvature scales," Image and Vision Computing, vol. 10, no. 8, pp. 557-564, 1992.

[11] J.A. Sethian, Level set Methods: evolving interfaces in computation geometry, fluid mechanics, computer vision, and materials science, Cambridge University Press, 1999.

[12] M.G. Linguraru, W.J. Richbourg, J. Liu, et al., "Tumor Burden Analysis from CT Data of Diseased Patients via Automated Liver and Tumor Segmentation," IEEE Transactions on Medical Imaging, vol. 31, no. 10, pp. 1965-1976, 2012.

[13] M.G. Linguraru, J.A. Sandberg, Z. Li, F. Shah, R.M. Summers, "Automated Segmentation and Quantification of Liver and Spleen from CT Images using Normalized Probabilistic Atlases and Enhancement Estimation," Medical Physics, vol. 37, no. 2, pp. 771-783, 2010 